\title{
Classification of Landmine-Like Metal Targets Using Wideband Electromagnetic Induction
}

\author{
Ping Gao, Student Member, IEEE, Leslie Collins, Member, IEEE, Philip M. Garber, Student Member, IEEE, \\ Norbert Geng, Member, IEEE, and Lawrence Carin, Senior Member, IEEE
}

\begin{abstract}
In our previous work, we have shown that the detectability of landmines can be improved dramatically by the careful application of signal detection theory to time-domain electromagnetic induction (EMI) data using a purely statistical approach. In this paper, classification of various metallic landmine-like targets via signal detection theory is investigated using a prototype wideband frequency-domain EMI sensor. An algorithm that incorporates both a theoretical model of the response of such a sensor and the uncertainties regarding the target/sensor orientation is developed. This allows the algorithms to be trained without an extensive data collection. The performance of this approach is evaluated using both simulated and experimental data. The results show that this approach affords substantial classification performance gains over a standard approach, which utilizes the signature obtained when the sensor is centered over the target and located at the mean expected target/sensor distance, and thus ignores the uncertainties inherent in the problem. On the average, a $60 \%$ improvement is obtained.
\end{abstract}

Index Terms-Bayes procedures, electromagnetic induction, object detection, pattern classification.

\section{INTRODUCTION}

A PERSISTENT problem with traditional narrowband EMI sensors involves not just detection of metal objects, but discrimination of targets from clutter. In most fielded sensors, the energy in the output of such sensors is calculated, and a decision regarding the presence or absence of a target is made using this statistic [1]. This approach leads to excessively large false alarm rates. When each piece of buried metal must be excavated in order to determine whether it is a target of interest, significant costs are incurred both due to lost time and costs associated with digging. The false alarm issue is particularly problematic in real world landmine-detection scenarios. In order to facilitate the discrimination of targets of interest from other pieces of metal, several modifications to traditional EMI sensors have been considered [1]-[8]. For instance, the late time EMI fields are characterized by an exponential decay in the time-domain [2], [3], [7], [8]. The decay rate has been used for target identification, because it strongly depends on the target conductivity, permeability, shape, and orientation. Alternatively, a promising approach is to operate the sensor in the frequency-domain by utilizing wideband excitation. The frequency dependence of the

Manuscript received November 23, 1998; revised June 15, 1999. This work was supported by the Army Research Office, Research Triangle Park, NC, under Grants DAAH04-96-1-0448 (Demining MURI) and DAAG55-98-1-0340.

The authors are with the Department of Electrical and Computer Engineering,

Duke University, Durham, NC 27708 USA (e-mail: lcollins@ee.duke.edu).

Publisher Item Identifier S 0196-2892(00)02849-7. induced fields excited by buried conducting targets can then be exploited by a detector.

A second problem that besets statistical algorithms is the need for adequate training data. A wide range of targets and clutter signatures must be obtained at all possible object/sensor orientations. Classically, this problem has been addressed by using data measured in the field [1], [9], [10]. However, a lack of sufficient training data can severely degrade performance [1], [11]. In general, it is difficult if not impossible to obtain such data for all possible object/sensor orientations. An alternative is to train the algorithms with data produced by computational models, which have only recently become general enough to consider such problems [8].

In this paper, we consider the problem in which we assume an object has been detected, and a decision as to "target" or "clutter" is required. In this approach, we use the complex frequency-dependent EMI response as a signature. A full-wave model is developed for the wideband EMI response of targets, specialized to the case of a body of revolution, thus obviating the need for training on field-collected data. Subsequently, a Bayesian classification algorithm is developed, which incorporates the wave model and the target/sensor position uncertainty. Substantial improvements are achieved via this approach over a processor, which ignores the orientation uncertainties.

This paper is organized as follows. In Section II, we describe a new prototype wideband frequency-domain EMI sensor, the GEM-3 [9]. In Section III, we discuss a model that calculates the wideband EMI responses. A model-based Bayesian approach for discriminating targets is discussed in Section IV. The process used to generate the simulated data and the experiment performed to collect the measured data are described in Section V. Next, the results from both simulated and measured data are shown. Finally, we summarize our major findings based on these results.

\section{SENSOR OVERVIEW}

When operating an EMI sensor in the frequency-domain, it has been shown that the frequency-dependent induced fields can differ significantly depending on the target shape and conductivity [12]. This variability may be exploited to enhance discrimination performance. Therefore, data from a prototype wideband EMI sensor, the GEM-3, developed by Geophex Ltd., was selected for this analysis. The validity of a numerical model that predicts the wideband EMI responses (discussed in the next section) can be tested using data collected with the GEM-3. Furthermore, a decision-theoretic discrimination algorithm can be applied to both simulated data generated based on the model 
predictions and real data measured with the sensor. In this section, the sensor operating principles are briefly described.

The design of the transmitting coils of the GEM-3, two concentric circular coils, results in a magnetic cavity in the center zone of the two coils, i.e., an area where the primary magnetic flux vanishes. A small receiving coil is located in this magnetic cavity [13]. Therefore, the receiver can sense a weak, secondary field returned from the earth and any buried objects. By using two transmitting coils connected in series, the coils carry the same amount of current. However, current flows in opposite directions. The magnetic cavity is created by carefully choosing the radius of the two coils and the number of turns of the coils. The sensor records the real and imaginary parts (in-phase and quadrature) of the induced complex voltage at the receiving coil, relative to that on the transmitting coils.

Instead of using a pulse excitation, such as that used by timedomain EMI systems, the transmitting coils of the wideband frequency-domain EMI sensor transmit a continuous, complex waveform consisting of multiple frequencies predefined by the operator [9], [13]. Thus, the sensor is only subject to the noise at the frequencies of interest, not within the whole frequency band, as is the case for time-domain EMI sensors. Therefore, frequency-domain EMI sensors can operate at much higher SNR's than time-domain systems. In addition to the improved SNR, theoretical calculations and experimental data have shown that the frequency-domain EMI signatures differ significantly across targets [12], which provides the underlying physical mechanisms important for discriminating, identifying, or classifying targets.

\section{MODEL FOR WIDEBAND FREQUENCY-DOMAIN EMI RESPONSES}

In this paper, a model-based Bayesian decision-theoretic approach is investigated to discriminate four manmade metal targets of different shapes, sizes, and metal types under conditions where the target/sensor relative position is unknown. In order to model the signature of these targets, a method of moment $(\mathrm{MoM})$ analysis is used to predict the theoretical response from the target [12], [14]. The calculation provides the theoretical induced voltage (magnitude and phase, or in-phase and quadrature components) for each target and frequency considered. Later in this paper, it is shown that by incorporating the model into the detector formulation, the classification performance is improved dramatically when the relative target/sensor position is uncertain, as it is in field operations.

We consider the fields induced by a highly (but not perfectly) conducting and/or permeable target in free space, due to EMI excitation at $\mathrm{kHz}$ frequencies. The problem is solved via a frequency-domain boundary-integral equation formulation. Moreover, to make such an analysis tractable, we specialize to a particular class of targets: those that can be modeled as a body of revolution (BOR) [15] (i.e., targets possessing rotational symmetry). Although here we consider near-field effects for metallic and ferrous targets, the general formulation is very similar to those used previously for far-zone scattering from low-loss dielectric targets [16], [17]. In particular, the problem is formulated in terms of the tangential electric $\mathbf{E}$ and magnetic $\mathbf{H}$ fields on the target surface or equivalently, in terms of electric and magnetic surface currents $\mathbf{J}=\mathbf{n} \times \mathbf{H}$ and $\mathbf{K}=\mathbf{E} \times \mathbf{n}$, respectively, where $\mathbf{n}$ is the outward unit normal. If $\mathbf{E}_{1}$ and $\mathbf{H}_{1}$ represent, respectively, the electric and magnetic fields inside the target, and $\mathbf{E}_{2}$ and $\mathbf{H}_{2}$ represent the "scattered" fields outside the target, boundary conditions at the interface yield the relationships (enforced at the boundary)

$$
\begin{aligned}
& \mathbf{n} \times\left[\mathfrak{L}_{1}^{E J}(-\mathbf{J})+\mathfrak{L}_{1}^{E K}(-\mathbf{K})\right] \\
& \quad=\mathbf{n} \times\left[\mathfrak{L}_{2}^{H J}(-\mathbf{J})+\mathfrak{L}_{2}^{E K}(-\mathbf{K})\right]+\mathbf{n} \times \mathbf{E}^{i} \\
& \mathbf{n} \times\left[\mathfrak{L}_{1}^{H J}(-\mathbf{J})+\mathfrak{L}_{1}^{H K}(-\mathbf{K})\right] \\
& \quad=\mathbf{n} \times\left[\mathfrak{L}_{2}^{H J}(-\mathbf{J})+\mathfrak{L}_{2}^{H K}(-\mathbf{K})\right]+\mathbf{n} \times \mathbf{H}^{i}
\end{aligned}
$$

where $\mathbf{E}^{i}$ and $\mathbf{H}^{i}$ represent the incident fields. The operators $\mathfrak{L}_{n}$ involve well-known manipulations of the homogeneousmedia Green's function [17]-[19] for medium parameters inside $(n=1)$ and outside $(n=2)$ the body. The problem therefore reduces to solving for $\mathbf{J}$ and $\mathbf{K}$ for particular incident fields $\mathbf{E}^{i}$ and $\mathbf{H}^{i}$. In the MoM solution for BOR [15]-[20], J, K, $\mathfrak{L}_{n}$, $\mathbf{E}^{i}$, and $\mathbf{H}^{i}$ are expanded in a Fourier series in the azimuthal variable $\phi$, and for each Fourier component, $\mathbf{J}$ and $\mathbf{K}$ are expanded in terms of one-dimensional (1-D) basis functions along the BOR generating arc (see Fig. 1). In this paper, we use subsectional basis functions and testing functions, as in [18]-[20]. The interested reader is referred to [18]-[20] for details concerning implementation of the general algorithm, while here we focus on issues of particular relevance to the EMI problem.

We first consider requirements concerning the subsectional-basis-function discretization of $\mathbf{J}$ and $\mathbf{K}$. For scattering from low-loss targets, it is well known that approximately ten basis functions are required per wavelength [17]. In such problems, this rule is applied to the smallest wavelength of interest in the problem, generally corresponding to the medium inside the target. For the highly conducting targets of interest here, the wavenumber inside the target approximately satisfies $k_{1}=(1-j) / \delta$, where $\delta$ is the skin depth. To sample the Green's function phase $\exp \left(-j k_{1} R\right)$ sufficiently, we require $\Delta t / \delta \ll 2 \pi$ and $\Delta t / \delta \ll 1$ (for the real and imaginary parts of $k_{1}$, respectively), where $\Delta t$ is the basis-function width. These constraints are usually sufficient to satisfy the outer region sampling requirements ( $k_{2}$ generally representing the free-space wavenumber). Extensive numerical experiments indicate that accurate results are obtained if $\Delta t<\delta / 3$.

As discussed above, for EMI applications, we are generally interested in current loop excitation, as distinguished from the plane-wave fields considered for radar problems. While the fields due to a current loop are well known [21], [22], we discuss how such are placed into the BOR framework, as well as appropriate approximations for the EMI problem. In particular, the incident fields are derived from the vector potential [21], [22]

$$
A_{\phi}(\rho, z) \approx \frac{\mu_{0} I a}{4 \pi} \int_{0}^{2 \pi} \frac{d \phi^{\prime} \cos \phi^{\prime}}{\sqrt{\rho^{2}+a^{2}+z^{2}-2 a \rho \cos \phi^{\prime}}}
$$

where the origin of the local cylindrical coordinate system $(\rho, \phi, z)$ is situated at the loop center, with axis parallel to $z$, and $I$ and $a$ are the loop current and radius, respectively. The 


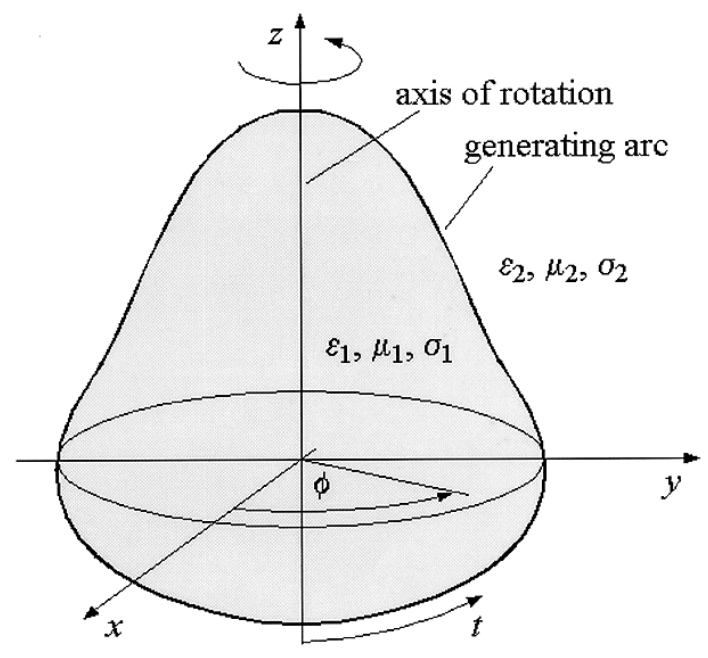

Fig. 1. Equivalent electric and magnetic surface currents for modeling electromagnetic interaction with a highly conducting and/or permeable body of revolution (BOR)

expression in (2) invokes a quasi-static approximation, since, at the wavelengths of interest (in air and soil), the electrical distance between the sensor and target is infinitesimal. Similar approximations can be used (but have not been here) with regard to the Green's function components in the air region $\left(\mathfrak{L}_{2}\right.$ in (1)), while the very high conductivity and/or permeability inside the target necessitates a rigorous analysis (i.e., a rigorous formulation of $\mathfrak{L}_{1}$ ). The incident electric and magnetic fields are readily computed as

$$
\begin{aligned}
H_{\rho}^{i}(\rho, z) & =-\frac{1}{\mu} \frac{\partial A_{\phi}}{\partial z}, \\
H_{z}^{i}(\rho, z) & =-\frac{1}{\mu \rho} \frac{\partial\left(\rho A_{\phi}\right)}{\partial \rho}, \\
E_{\rho}^{i}(\rho, z) & \approx-j \omega A_{\phi}
\end{aligned}
$$

and these fields are finally expressed in terms of complete elliptical integrals. If the loop axis and the BOR axis are aligned, the fields in (3) can be applied directly to the BOR MoM solution, and only the lowest-order Fourier series mode is excited (reflecting azimuthal symmetry). If the axes are not aligned, a Fourier series representation of the incident tangential fields on the BOR surface is generally required (with the BOR problem solved separately using the incident fields from each such mode [15]-[18]). While the Fourier components for plane-wave incidence can be expressed in closed form [15], we have not found such a simple representation for the loop-induced fields. Therefore, in the general case, we must numerically determine the Fourier coefficients. For example

$$
\hat{H}_{m t}(t)=\frac{1}{2 \pi} \int_{0}^{2 \pi} H_{t}^{i}(t, \phi) \exp (-j m \phi) d \phi
$$

where $H_{t}^{i}$ represents the incident magnetic field along the generating arc (Fig. 1), and $(t, \phi)$ represents the local BOR coordinate system. Thus, while the space domain fields for the loop can be expressed in closed form, the requisite Fourier components are evaluated numerically. However, the incident fields are gener- ally slowly varying in $\phi$ and integrals of the type in (4) do not present a significant numerical challenge.

Before proceeding to a comparison of theoretical and measured results, we note that the EMI fields induced by a conducting and/or ferrous target are generally measured in the near zone. Therefore, when calculating the induced fields, we cannot invoke the simplifying far zone approximation generally used for radar-scattering problems [15], [17], [23]. We therefore calculate the EMI "scattered" fields via a rigorous convolution of the calculated currents $\mathbf{J}$ and $\mathbf{K}$ with the free-space Green's function (e.g., with $\mathfrak{L}_{2}$ ), performing integrals similar to those used in calculating the components of the MoM impedance matrix. Additionally, we note that an actual EMI sensor does not measure the induced fields, but rather the electromotive force induced on a sensing current loop. To calculate such, we have used appropriate magnetic field components, integrated over the aperture of the sensing loop, to generate a theoretical induced voltage for each target and frequency considered.

Using data collected from a prototype wideband frequencydomain EMI sensor, the GEM-3, the effectiveness of the numeric model is tested. A comparison of the theoretical model and measurements is shown in Section V-B.

The simulation outputs from the model can be used to calibrate the frequency-domain EMI sensor. Let $c(\omega)$ represent the calibration constant for frequency $\omega$, the $K \times 1$ vector M represent a set of measurements obtained at several $(K)$ positions, and the $K \times 1$ vector $\mathbf{B}$ represent model outputs for the same target and positions. We have the relation that $\mathbf{B} c(\omega)=\mathbf{M}$, and a least-squares method is used to obtain the calibration constants as a function of frequency.

\section{Forward Model-BASED BAYESIAN Classifier FORMULATION}

In a real-world classification scenario, the uncertainty inherent in the sensor output is not only due to additive noise, but also to the fact that the relative position between the sensor and the target is unknown at the point when the measurements are obtained. In this work, we investigate the classification performance of a Bayesian classifier that incorporates modeled wideband EMI signatures as well as position uncertainties and compare its performance to an approach that ignores these uncertainties and assumes the target is at a fixed position corresponding to the mean assumed position.

In this paper, we consider the task of classifying data from one of four known metal objects. It is always true that one of the objects is present, and our goal is to decide which object is present. In real world situations such as landmine detection, it is often the case that a metal object can be located. The task is then to determine whether it is a target or a clutter object. In this case, a library of targets of interest can be established and typical clutter can also be modeled. Alternatively, a statistical model could be imposed for clutter based on localized measurements and the target models can be used as is described here. Thus, this approach can also be applied to an extended set of objects in practice.

In this work, four metal objects are considered (a more detailed description can be found in Section V). Signals used 
to measure classification performance are either the modeled wideband frequency-domain EMI responses or measured EMI responses from the GEM-3. Since any sensor is subject to noise, which is usually assumed to follow a Gaussian distribution, the distribution of the sensor outputs (obtained data set of discrete frequencies) while the target/sensor is at a known height and horizontal position, is a Gaussian random vector. The mean of this response is the theoretical response, and the variance is equal to that of the additive noise. Let $H_{i}$ represent the hypothesis that the $i$ th target is present, where $i=1,2,3,4$. The received data from the $i$ th target at a known position can be modeled as

$$
r_{i j}=A_{i j}+n_{j}
$$

where $j$ corresponds to the discrete frequencies of interest, $j=$ $1,2, \ldots, N, r_{i j}$ is the received data from the sensor, $A_{i j}$ is the predicted response obtained from the model (as described in detail in Section III, the model can calculate the theoretical frequency-domain EMI responses for a well specified BOR object at a known position) for the $i$ th target at the $j$ th frequency at a known depth and horizontal position relative to the center of the sensor, and $n_{j}$ is Gaussian noise with zero mean and variance of $\sigma_{n_{j}}^{2}$. We assume that $n_{j}$ 's are independent. However, their variance is a function of frequency. Let $q_{i}$ represent the a priori probability that hypothesis $H_{i}$ is true. We further assume that the cost of a correct decision is zero, and the cost of any wrong decision equals 1 . Bayes' solution for this classification problem [24], [25] is to decide that $H_{i}$ is true if

$$
\frac{p\left(H_{i} \mid r\right)}{p\left(H_{k} \mid r\right)}=\frac{q_{i} p\left(r \mid H_{i}\right)}{q_{k} p\left(r \mid H_{k}\right)}>1
$$

is satisfied for any $k \neq i$. Here $p\left(H_{i} \mid r\right)$ is the a posteriori distribution or discriminant function [26], $p\left(r \mid H_{i}\right)$ is the probability density or likelihood function of data $r$ given $H_{i}$, and $r$ is the received data from the sensor. Assuming the magnitude and the phase of the frequency response are independent, $\mathbf{r}$ is a vector containing both the magnitude and phase information. Therefore, when the sampled data $r$ is received, we decide in favor of hypothesis $H_{i}$, where

$$
q_{i} p\left(\mathbf{r} \mid H_{i}\right)=\max _{k}\left\{q_{k} p\left(\mathrm{r} \mid H_{k}\right)\right\} \quad k=1,2,3,4
$$

Thus, we decide in favor of a hypothesis that has the largest a posteriori probability or largest discriminant function at $r$ among all four possible pdf's. Since we usually have no $a$ priori knowledge of $q_{i}$ (in other words, we do not know the probability that a particular target is going to be present), an equal probability assumption for each target is made (i.e., $q_{i}=1 / 4$ ). Based on the uniform priori on $q_{i}$, (7) can be further understood as seeking a hypothesis that provides the maximum likelihood among the four possible values. Thus, it can also be referred to as a maximum likelihood (ML) classifier. Since any monotonically increasing function of $p\left(H_{i} \mid \mathbf{r}\right)$ is also a valid discriminant function [26], an alternative discriminant function based on the above assumptions is

$$
\begin{aligned}
& p\left(\mathbf{r} \mid H_{i}\right) \\
& \quad=(2 \pi)^{-N}|\Sigma|^{-1 / 2} \exp \left[-\frac{1}{2}\left(\mathbf{r}-\mathbf{A}_{i}\right)^{T} \Sigma^{-1}\left(\mathbf{r}-\mathbf{A}_{i}\right)\right](8)
\end{aligned}
$$

TABLE I

PROBABILITY OF CORRECT CLASSIFICATION OF THE OPTIMAL CLASSIFIER WhEN TARgets are at a FiXed KNOWN Position as the NoISE VARIANCE IS INCREASED FROM $\sigma_{n}^{2}$ TO $2^{9} \sigma_{n}^{2}$

\begin{tabular}{c|c|c|c|c}
\hline \multirow{2}{*}{ NOISE VARIANCE } & \multicolumn{4}{|c}{ PROBABILITY OF CORRECT CLASSIFICATION } \\
\cline { 2 - 5 } & TARGET 1 & TARGET 2 & TARGET 3 & TARGET 4 \\
\hline$\sigma_{n}{ }^{2}$ & 1.0000 & 1.0000 & 1.0000 & 1.0000 \\
\hline $2 \sigma_{n}{ }^{2}$ & 1.0000 & 1.0000 & 1.0000 & 1.0000 \\
\hline $2^{2} \sigma_{n}^{2}$ & 0.9993 & 1.0000 & 0.9990 & 1.0000 \\
\hline $2^{3} \sigma_{n}{ }^{2}$ & 0.9862 & 1.0000 & 0.9877 & 1.0000 \\
\hline $2^{4} \sigma_{n}^{2}$ & 0.9365 & 1.0000 & 0.9394 & 1.0000 \\
\hline $2^{5} \sigma_{n}{ }^{2}$ & 0.8604 & 1.0000 & 0.8640 & 1.0000 \\
\hline $2^{6} \sigma_{n}^{2}$ & 0.7809 & 0.9977 & 0.7747 & 1.0000 \\
\hline $2^{7} \sigma_{n}{ }^{2}$ & 0.7134 & 0.9791 & 0.6854 & 1.0000 \\
\hline $2^{8} \sigma_{n}{ }^{2}$ & 0.6545 & 0.9254 & 0.5756 & 0.9981 \\
\hline $2^{9} \sigma_{n}^{2}$ & 0.6101 & 0.8552 & 0.4554 & 0.9827 \\
\hline
\end{tabular}

where $N$ is the total number of frequencies used, $\mathbf{r}$ and $\mathbf{A}_{i}$ are $2 N$ by 1 vectors, and $\Sigma$ is the covariance matrix of $\mathrm{r}$. Given the assumptions on the noise process, $\Sigma$ is a diagonal matrix with $\sigma_{n_{j}}^{2}$ on the $j$ th diagonal, where $j$ corresponds to frequency. Since the coefficient of the exponential term of (8) is the same for all the hypotheses, it can be neglected. After taking the logarithm, the alternative discriminant function simplifies to

$$
\log p^{\prime}\left(\mathbf{r} \mid H_{i}\right)=-\left(\mathbf{r}-\mathbf{A}_{i}\right)^{T} \Sigma^{-1}\left(\mathbf{r}-\mathbf{A}_{i}\right)
$$

where $\left(\mathbf{r}-\mathbf{A}_{i}\right)^{T} \Sigma^{-1}\left(\mathbf{r}-\mathbf{A}_{i}\right)$ is often referred to as the Mahalanobis distance from $r$ to $\mathbf{A}_{i}$ [26]. If $\Sigma$ is a diagonal matrix with each diagonal element $\sigma_{n_{j}}^{2}$, (9) can be expressed as

$$
\log p^{\prime \prime}\left(\mathbf{r} \mid H_{i}\right)=-\sum_{j=1}^{2 N}\left(r_{j}-A_{i j}\right)^{2} / \sigma_{n_{j}}^{2}
$$

The discriminant function obtained above [(10)] is valid if the height and horizontal position of the object are both known, and the noise is assumed to be independent at each frequency. This solution is optimal only under the assumptions that all the parameters are known, and the sensor is subject only to Gaussian noise. This formulation differs from a bank of matched filters since the noise is not identically distributed and the variance of the noise is a function of frequency, and the signals are not of equal energy. These two facts result in a formulation, which is similar to, but not identical to, the traditional matched filter $r^{T} A_{i}$, which is the result of $i . i . d$ additive white Gaussian noise [25], [27].

The performance of the classifier given by (10) is a function of the noise variance and the modeled response. Table I lists the theoretical performance of the classifier as the noise variance is increased from $\sigma_{n}^{2}$ to $2^{9} \sigma_{n}^{2}$, where $\sigma_{n}^{2}$ is a vector that contains the noise variance of the magnitude and phase as a function of frequency obtained from experimental data (see Section. V-B). As expected, an increase in the noise variance results in a decrease in the classification performance. This analysis provides 
insight into how the classification performance is affected by the sensor noise. Once the sensor is manufactured and well calibrated, the sensor noise cannot be changed artificially. Thus, only simulated data was used to illustrate this effect.

A more realistic assumption for the classification problem is that the height and horizontal position are uncertain, since the exact sensor position where measurements are obtained relative to the underground objects is unknown in practice. In this case, the previously derived processor [as in (10)], which assumed a "fixed" target/sensor orientation, is not the optimal solution. Hence, in order to obtain the optimal discriminant function for the received data, the effect of these random factors must be integrated out, i.e.,

$$
p\left(\mathbf{r} \mid H_{i}\right)=\iiint p\left(\mathbf{r} \mid H_{i}, h, x, y\right) p(h) p(x, y) d h d x d y
$$

where $h$ represents the height of the sensor from the target, $x$ and $y$ represent the horizontal position of the sensor relative to the center of the target, $p(h)$ and $p(x, y)$ are the a priori distributions of the position factors, and

$$
\begin{aligned}
p\left(\mathbf{r} \mid H_{i}, h, x, y\right) & \\
= & \frac{1}{(2 \pi)^{N}|\Sigma|^{1 / 2}} \exp \left(-\frac{1}{2}\left(\mathbf{r}-\mathbf{A}_{i}(h, x, y)\right)^{T}\right. \\
& \left.\times \Sigma^{-1}\left(\mathbf{r} \mathbf{A}_{i}(h, x, y)\right)\right)
\end{aligned}
$$

where $\mathbf{A}_{i}(h, x, y)$ is the model prediction (described in detail in Section III) of the $i$ th target response when it is located at the position $(h, x, y)$ relative to the sensor. The model predicts the theoretical frequency-domain EMI response as a function of constitutive parameters, exact dimensions of the object and the horizontal and vertical distance from the center of sensor to that of the object. Monte Carlo integration was implemented in order to calculate the integral in (11).

\section{Simulated AND EXPERIMENTAL DATA}

The performance of both the fixed-position processor (10) and the optimal classifier (11) is investigated by using both simulations and measurements for the GEM-3 sensor. In this section, the method used to generate simulated data, the experimental design, and the methods used to take the measurements are described.

Four metal targets are considered for both the simulations and experimental measurements: an aluminum barbell, an aluminum disk, a thick brass disk, and a thin brass disk. The dimensions of these targets are as follows. The diameter of each of the targets is $5.08 \mathrm{~cm}$. The heights of the targets are $2.897 \mathrm{~cm}, 2.667$ $\mathrm{cm}, 2.34 \mathrm{~cm}$, and $0.3175 \mathrm{~cm}$ for the aluminum bar-bell, the aluminum disk, the thick and the thin brass disk, respectively. The response from a target depends on the constitutive parameters, geometry of the target, as well as the horizontal and vertical distance from the center of the sensor to that of the target. In the calculations, 21 linearly spaced frequencies were chosen, ranging from $3990 \mathrm{~Hz}$ to $23970 \mathrm{~Hz}$. These frequencies are within the range that the GEM-3 operates.

\section{A. Simulations}

In order to test whether the classification performance is improved by incorporating the model into the classification formulation, several cases were considered. These cases are:

1) fixed position;

2) random height but fixed horizontal position;

3) random horizontal position but fixed height;

4) both height and horizontal position random.

It is assumed that the distribution of the height, $h$, follows a Gaussian distribution with a mean of $20 \mathrm{~cm}$ and variance of $1.53^{2} \mathrm{~cm}^{2}$ and the horizontal position is uniformly distributed in a $20 \mathrm{~cm}$ by $20 \mathrm{~cm}$ square. To generate the simulated data we specify the constitutive parameters and the dimensions of the target, then generate 10000 random sets of height $(h)$ and horizontal position $(x, y)$, which follow the distributions described above. Based on these parameters, the wideband EMI response is calculated by the model for each set of $h, x$, and $y$. After obtaining the theoretical responses of each target at all the specified positions (which are used in the formulation of the detector), Gaussian noise is added to the theoretical responses to create the simulated data set. Both the processor, which assumes a fixed target/sensor orientation (10), and the optimal classifier (11) are then applied to these data. Results of these classifiers are discussed in Section VI.

\section{B. Measured Data}

Using synthetic data to evaluate the performance of the classifier provides useful insight regarding performance bounds, but limiting the analysis to simulated data is not sufficient. Therefore, measurements of the wideband frequency-domain EMI response from the four metal targets were taken using the GEM-3 in order to evaluate the improvement of the classification algorithm in a more realistic scenario. First we consider whether data taken from objects in air is comparable to data obtained when the objects are buried in soil. Figs. 2 and 3 provide the wideband frequency-domain EMI responses measured using the GEM-3 from two metal landmines, a Valmara (an antipersonnel metal landmine) and a VS50 (an antipersonnel metal mine), in air and in North Carolina clay soil (buried 1 in below the surface). These figures indicate that the soil effects can be neglected at least for large metal objects. Therefore, the measured data used to evaluate the performance of various classification techniques was taken in free space.

The experimental set up is as follows. The GEM-3 was mounted on a wooden rack with the sensor head, approximately $1.8 \mathrm{~m}$ above the wooden base of the platform. Both rack and platform contained no metal parts. The rack assembly allows placement of a target on a wooden shelf at various distances beneath the sensor head.

First, in order to obtain an estimate of the noise variance associated with the sensor, $\sigma_{n_{j}}^{2}, 100$ measurements were taken with the sensor at a fixed position and no target present. We refer to an individual measurement taken without a target present as a background response. This response is subtracted from the responses measured with the target present to estimate the response due to the target alone. Fig. 4 shows a typical plot of the background 


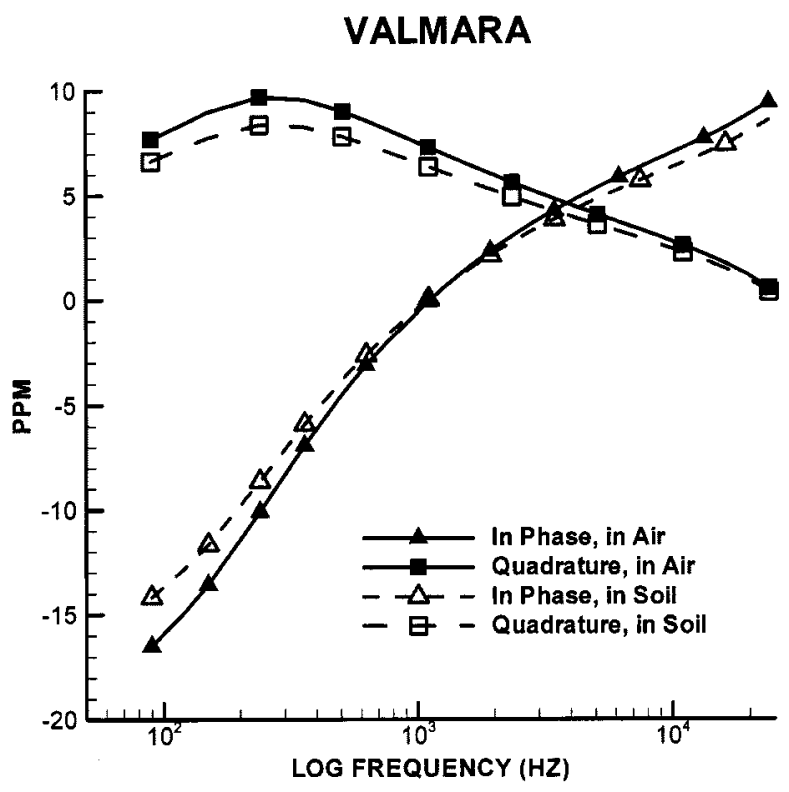

Fig. 2. Wideband frequency-domain response of a Valmara in free space and buried 1 in below the surface of the ground in North Carolina clay soil. The units "ppm" reflect the sensor output multiplied by $10^{6}$.

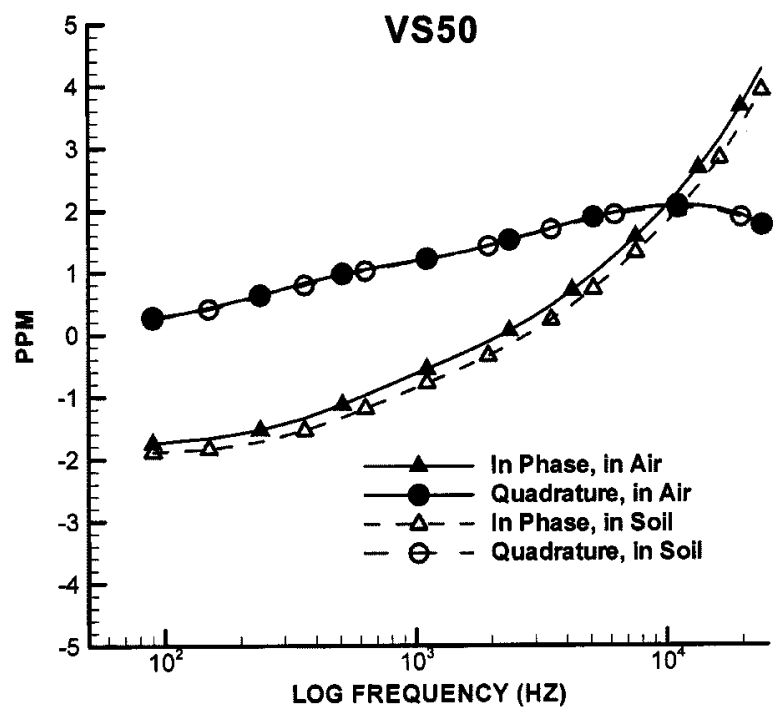

Fig. 3. Wideband frequency-domain response of a VS50 in free space and buried 1 in below the surface of the ground in North Carolina clay soil. The units "ppm" reflect the sensor output multiplied by $10^{6}$.

response. The background response is not the same at all frequencies, and the noise variance is also a function of frequency. Table II lists the variance of the noise for the magnitude and phase of the complex response and the ratio between the mean value of the response and the standard deviation of the noise as a function of frequency, respectively. These estimates were used in the classifier given by (10) and (11).

As described in Section III, to calibrate the sensor, measurements for the four targets were taken so the calibration coefficients could be calculated. Each target was placed beneath the center of the sensor head at distances of $17 \mathrm{~cm}, 19 \mathrm{~cm}, 20 \mathrm{~cm}$, $21 \mathrm{~cm}$, and $23 \mathrm{~cm}$. Using these 20 measurements, calibration coefficients were calculated by the least-squares method. Fig. 5

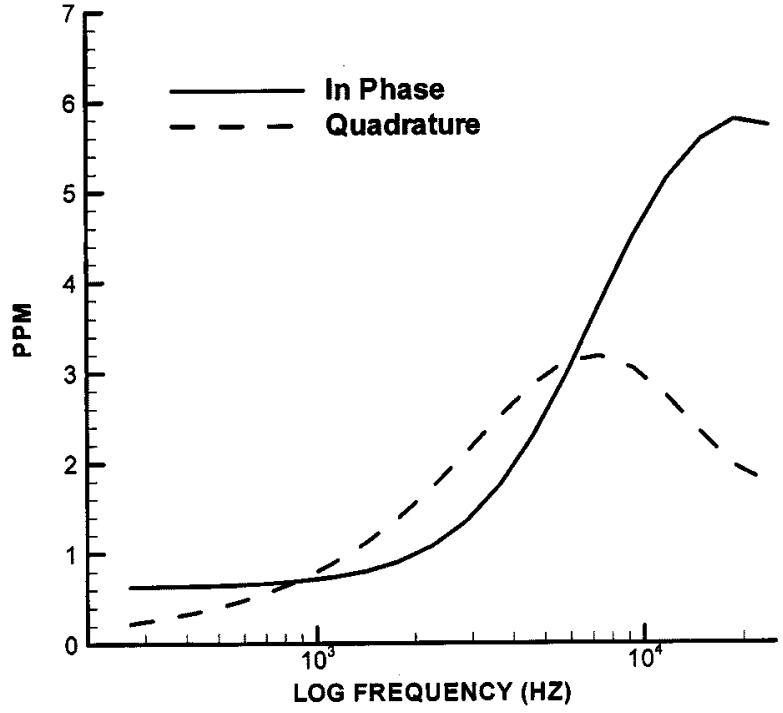

Fig. 4. Wideband frequency-domain response from Earth without the presence of any targets or background response. The units "ppm" reflect the sensor output multiplied by $10^{6}$.

shows the comparison of the theoretical model predictions and the measurements. As has been noted previously, the model predicts the GEM-3 response well [12].

To obtain the data used to evaluate algorithm performance, measurements were taken from each target at seven heights from $17 \mathrm{~cm}$ to $23 \mathrm{~cm}$ in $1 \mathrm{~cm}$ increments. The distribution of height is assumed to be Gaussian with a mean of $20 \mathrm{~cm}$ and a variance of $1.53^{2}$ for the simulations. At each height, between 11 and 36 measurements were taken. The exact count was calculated based on the assumed distribution. At each height, the position of each measurement is uniformly distributed within a $20 \mathrm{~cm} \times 20 \mathrm{~cm}$ square. For each target, there were a total of 328 measurements taken. These data were not used to train the algorithm, only to evaluate performance.

\section{RESULTS}

We exploit Bayesian decision theory to formulate an optimal classifier to discriminate these targets. In order to show the improvement of the optimal classifier, the performance of a processor that assumes a fixed target/sensor orientation was also evaluated. It was assumed that the sensor is subject to a small amount of additive Gaussian noise. This assumption is verified by the experimental data (see Section V-B). The performance of these classifiers, shown in this section, was evaluated using both synthetic data and experimental measurements.

\section{A. Simulation Results}

1) Fixed Height and Horizontal Position: First, the case where all the position parameters are known exactly is considered. The model of each target at the same position and all desired frequencies is calculated. Then, by adding Gaussian random noise with zero mean and variance obtained based on the experimental data (see Table II), 10000 realizations of simulated data for each target are generated. The decision of which target is present is made based on (7) by using the 
TABLE II

Variance of the Background NoISE and the Ratio of the Mean of the Response and Its Standard Derivation as a Function of FREQUENCY FOR THE MAGNITUDE AND PHASE COMPONENTS, RESPECTIVELY

\begin{tabular}{c|c|c|c|c}
\hline \multirow{2}{*}{ Frequency (Hz) } & \multirow{2}{*}{$\sigma_{\text {magntude }}^{2}$} & mean(magnitude) & $\sigma_{\text {phase }}$ & $\frac{\text { mean(phase) }}{n}$ \\
\cline { 3 - 5 } & & $\sigma_{\text {mgantude }}$ & & $\sigma_{\text {hhase }}$ \\
\hline 3,990 & $8.25 \mathrm{E}-05$ & $3.62 \mathrm{E}+02$ & $8.86 \mathrm{E}-02$ & $1.74 \mathrm{E}+02$ \\
\hline 5,010 & $8.99 \mathrm{E}-05$ & $4.12 \mathrm{E}+02$ & $5.55 \mathrm{E}-02$ & $2.00 \mathrm{E}+02$ \\
\hline 5,970 & $9.41 \mathrm{E}-05$ & $4.52 \mathrm{E}+02$ & $3.91 \mathrm{E}-02$ & $2.16 \mathrm{E}+02$ \\
\hline 6,990 & $9.47 \mathrm{E}-05$ & $4.95 \mathrm{E}+02$ & $2.83 \mathrm{E}-02$ & $2.27 \mathrm{E}+02$ \\
\hline 8,010 & $8.96 \mathrm{E}-05$ & $5.46 \mathrm{E}+02$ & $2.19 \mathrm{E}-02$ & $2.31 \mathrm{E}+02$ \\
\hline 8,970 & $8.35 \mathrm{E}-05$ & $5.96 \mathrm{E}+02$ & $1.77 \mathrm{E}-02$ & $2.31 \mathrm{E}+02$ \\
\hline 9,990 & $7.47 \mathrm{E}-05$ & $6.58 \mathrm{E}+02$ & $1.44 \mathrm{E}-02$ & $2.28 \mathrm{E}+02$ \\
\hline 11,010 & $6.57 \mathrm{E}-05$ & $7.27 \mathrm{E}+02$ & $1.21 \mathrm{E}-02$ & $2.22 \mathrm{E}+02$ \\
\hline 11,970 & $5.73 \mathrm{E}-05$ & $8.00 \mathrm{E}+02$ & $1.05 \mathrm{E}-02$ & $2.14 \mathrm{E}+02$ \\
\hline 12,990 & $5.44 \mathrm{E}-05$ & $8.42 \mathrm{E}+02$ & $8.99 \mathrm{E}-03$ & $2.05 \mathrm{E}+02$ \\
\hline 14,010 & $5.70 \mathrm{E}-05$ & $8.40 \mathrm{E}+02$ & $8.05 \mathrm{E}-03$ & $1.92 \mathrm{E}+02$ \\
\hline 14,970 & $6.23 \mathrm{E}-05$ & $8.18 \mathrm{E}+02$ & $7.20 \mathrm{E}-03$ & $1.80 \mathrm{E}+02$ \\
\hline 15,990 & $9.25 \mathrm{E}-05$ & $6.82 \mathrm{E}+02$ & $6.23 \mathrm{E}-03$ & $1.70 \mathrm{E}+02$ \\
\hline 16,950 & $1.11 \mathrm{E}-04$ & $6.31 \mathrm{E}+02$ & $5.14 \mathrm{E}-03$ & $1.64 \mathrm{E}+02$ \\
\hline 17,970 & $1.55 \mathrm{E}-04$ & $5.42 \mathrm{E}+02$ & $4.87 \mathrm{E}-03$ & $1.46 \mathrm{E}+02$ \\
\hline 18,990 & $1.49 \mathrm{E}-04$ & $5.62 \mathrm{E}+02$ & $9.35 \mathrm{E}-03$ & $9.00 \mathrm{E}+01$ \\
\hline 19,950 & $1.63 \mathrm{E}-04$ & $5.40 \mathrm{E}+02$ & $1.39 \mathrm{E}-02$ & $6.25 \mathrm{E}+01$ \\
\hline 20,970 & $7.26 \mathrm{E}-04$ & $2.60 \mathrm{E}+02$ & $3.64 \mathrm{E}-03$ & $1.01 \mathrm{E}+02$ \\
\hline 21,950 & $3.90 \mathrm{E}-04$ & $3.58 \mathrm{E}+02$ & $9.92 \mathrm{E}-03$ & $4.99 \mathrm{E}+01$ \\
\hline 22,950 & $7.81 \mathrm{E}-04$ & $2.58 \mathrm{E}+02$ & $9.23 \mathrm{E}-03$ & $3.99 \mathrm{E}+01$ \\
\hline 23,970 & $1.21 \mathrm{E}-03$ & $2.10 \mathrm{E}+02$ & $8.32 \mathrm{E}-03$ & $3.10 \mathrm{E}+01$ \\
\hline
\end{tabular}

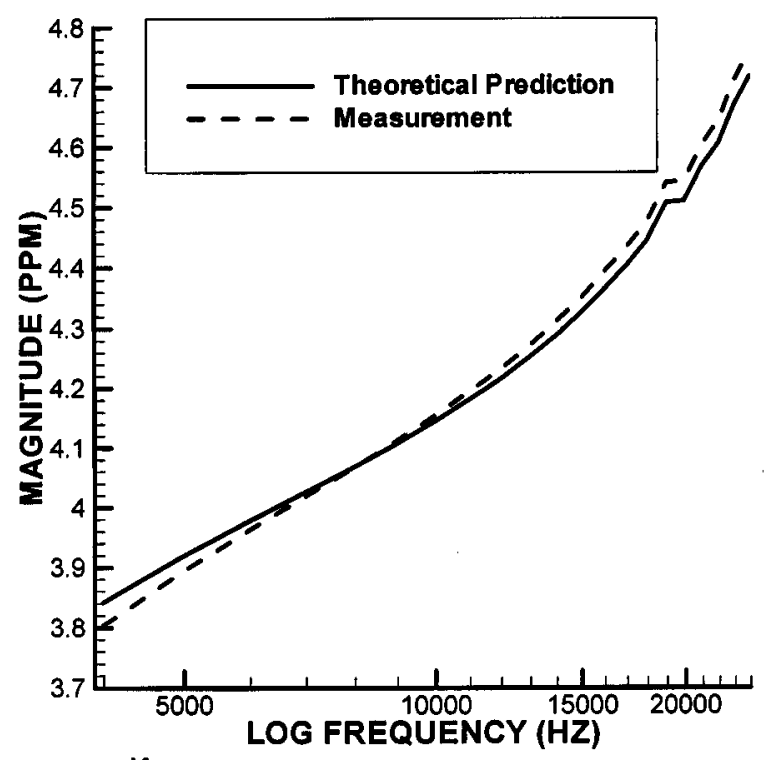

Fig. 5. Comparison of measurements and theoretical predictions for the thin brass disk when the distance from the target to the sensor is $20 \mathrm{~cm}$.

processor expressed in (10), which is optimal for this case. Because of the fact that the wideband EMI signature of these targets is significantly different [12] and the experimentally derived $\sigma_{n}^{2}$ 's are low, the performance is perfect.

2) Height Uncertain, Fixed Horizontal Position: Next, the case where only the height of the sensor from the target is unknown and the target is located under the center of the sensor is considered. This situation occurs in a real detection scenario when the sensor operator can accurately center the sensor, but the burial depth of the mine is unknown. The height of the sensor was modeled as a Gaussian distributed random variable with a mean of $20 \mathrm{~cm}$ and a variance of $1.53^{2} \mathrm{~cm}^{2}$. Fig. 6 shows the performance of a processor that assumed a fixed target/sensor orientation along with that of the optimal classifier. For the former, it is assumed the target is at the mean height of $20 \mathrm{~cm}$. Clearly, substantial improvements in classification performance are achieved by the optimal classifier over a processor that assumes a fixed target/sensor orientation. This performance is achieved for a relatively small level of uncertainty in the height. The average performance improvement is over $70 \%$.

3) Horizontal Position Uncertain, Fixed Height: Thirdly, we simulate the case where horizontal position is uncertain. It is assumed that the sensor is located at a known, fixed height. Because the exact positions of mines are unknown to the sensor operator during detection, we assumed a uniform distribution in the horizontal plane. Fig. 7 shows the simulation results of the processor that assumes a fixed target/sensor orientation and the optimal classifier when the horizontal positions of targets are uniformly distributed. For the former, it was assumed that the target was at the mean horizontal position and was directly under the sensor. Again, the performance of the optimal classifier is substantially better than that of the processor, which ignores the target/sensor orientation uncertainty. It improves on average by $60 \%$. 


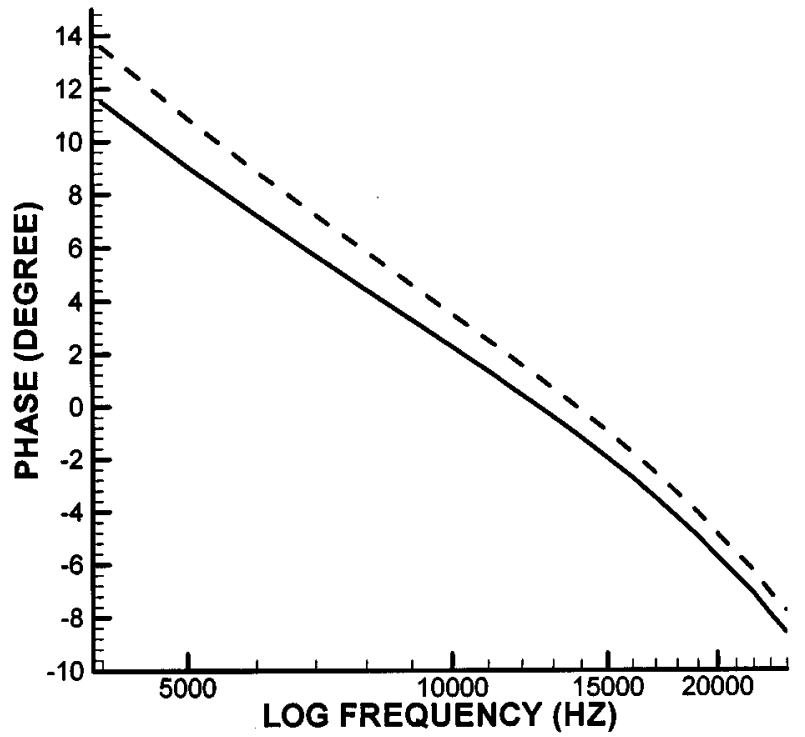

Fig. 6. Comparison of the processor, which ignores target/sensor orientation uncertainty ("fixed position" processor) and the optimal processor under uncertain height, fixed-horizontal position conditions for simulated data.

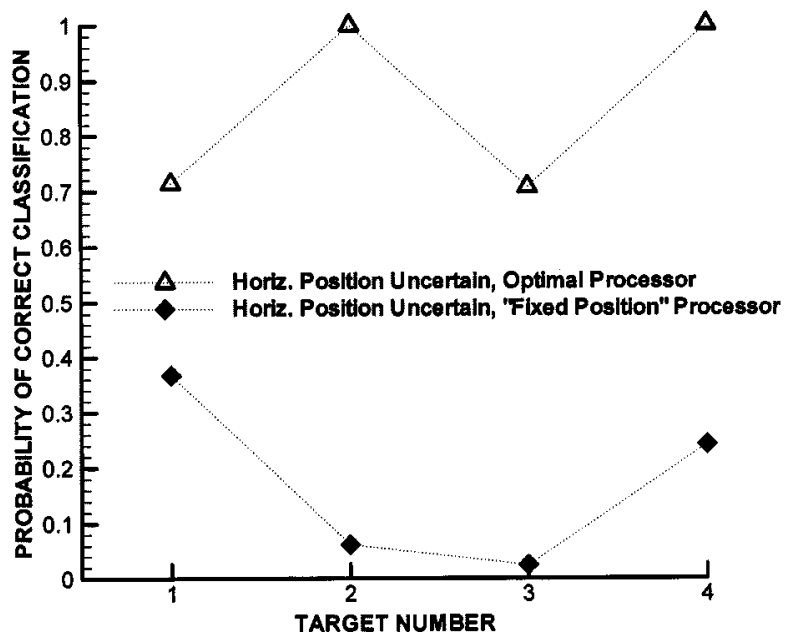

Fig. 7. Comparison of the "fixed position" processor and optimal processor performance under the uncertain horizontal position, but fixed height condition for simulated data.

4) Both Height and Horizontal Position Uncertain: In the final simulation, both height and horizontal position are uncertain. The height is assumed to follow a Gaussian distribution with mean of $20 \mathrm{~cm}$ and variance of $1.53^{2}$. The horizontal position follows a uniform distribution (within a $20 \mathrm{~cm} \times 20 \mathrm{~cm}$ square). Fig. 8 illustrates the performance of the two processors. The "fixed orientation" processor assumes that the target is located at the mean height and horizontal position. Performance improves under these conditions by an average of $70 \%$ over that of the "fixed position" processor. The results in Figs. 6-8 indicate that for the fixed position processor the performance becomes progressively worse as the position uncertainty increases. Clearly, incorporating the uncertainty of these environmental parameters into the processor affords a significant performance gain over a processor, which ignores this uncertainty.

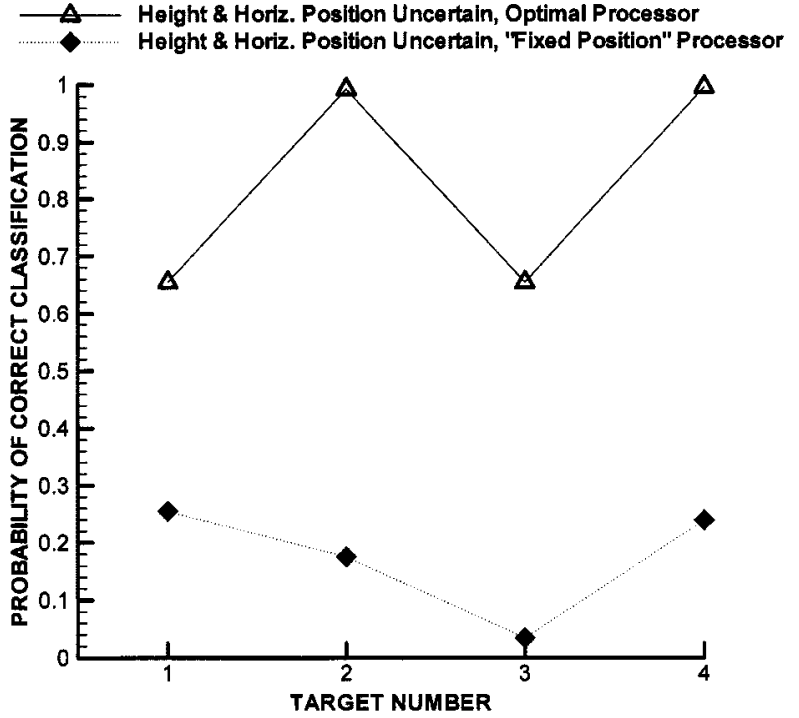

Fig. 8. Comparison of the "fixed position" processor and optimal processor performance when both height and horizontal position are uncertain for simulated data.

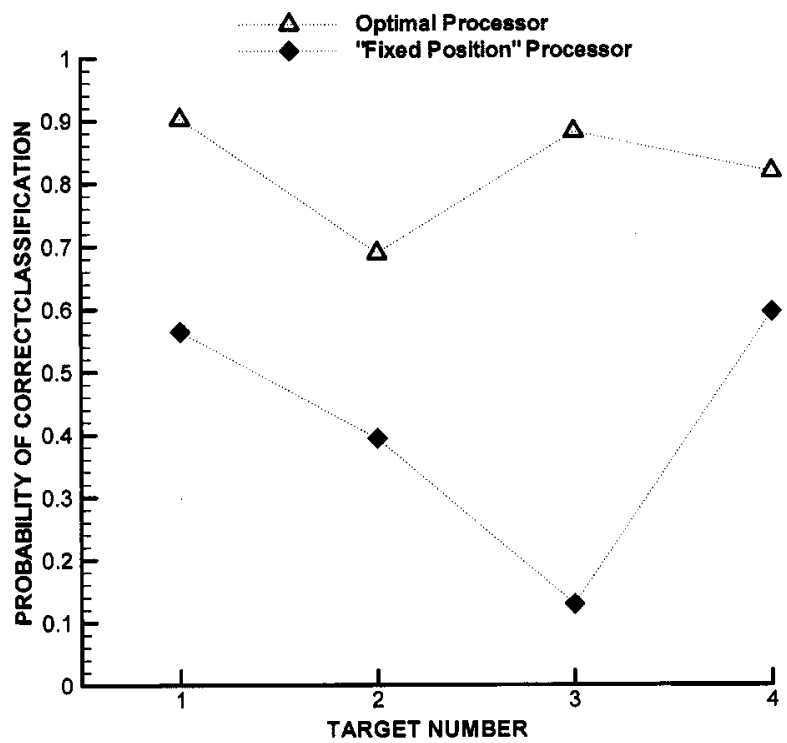

Fig. 9. Comparison of the "fixed position" processor and optimal processor performance under the condition of both height and horizontal position unknown for measured data.

\section{B. Experimental Data}

Simulations have shown that significant performance improvements can be achieved when the position uncertainty is incorporated into the classifier. To verify this result, measured data were collected using the GEM-3, as described in Section V-B. In this section, the results of implementing these processors using the measured data are shown.

The same two signal processing algorithms that were applied to simulated data: a fixed position processor, which assumes each target at the mean position, and the optimal classifier, which incorporates the position uncertainty into the processor, were applied to the experimental data. Fig. 9 illustrates the 
performance achieved by each of these two algorithms. Clearly, better performance is achieved by the optimal processor. Performance improves on the average by $60 \%$. This improvement, obtained on the measured data, is consistent with that observed in the simulated data set.

\section{CONCLUSION}

In this paper, we utilize a Bayesian decision-theoretic approach to classify metal targets using wideband EMI data. Four manmade metal targets were used. Results from both simulation and measured data, shown in Section V, indicate that incorporating the uncertainty associated with the target/sensor relative position into the processor affords a significant performance gain over a processor that matches to the predicted response at the mean expected target position. It is also noted that, as expected, under conditions of uncertainty the performance of both the fixed orientation processor and the optimal processors drops compared to that of the signal-known-exactly case. Though the optimal classifier can improve performance under uncertain conditions over processors that ignore the uncertainties, it will never achieve the performance obtained when no uncertainty is present.

As expected, simulations have shown that the SNR's of the signal affect the performance of a classifier. Since frequency-domain systems can achieve high SNR's compared to time-domain EMI systems, essentially, it will improve the classification performance.

Our work indicates that we can effectively discriminate different metal targets using wideband EMI signals by incorporating an accurate physical model and models of the uncertainty regarding environmental parameters into the classifier. Performance can be dramatically improved over the standard approach, which ignores environmental uncertainty. In addition, extensive libraries of target signatures do not have to be measured experimentally in order to train the classifier.

This technique can be extended to apply to other applications such as landmine detection and unexploded ordnance (UXO) detection, since in these applications, targets of interests need to be discriminated from metallic clutter in order to reduce false alarm rates. The standard algorithms for these applications, such as matched filters, do not take the uncertainties associated with the target/sensor orientation into account, and only partially exploited the underlying physical nature of the outputs from the sensor. The work shown in this paper provides a promising technique, which integrates both the uncertainties associated with target/sensor orientation and a forward model exploiting the physical signature of wideband frequency-domain EMI response. By developing a model for other signals and sensor modalities, this algorithm can be further applied to other applications that require classification of different targets.

\section{ACKNOWLEDGMENT}

The authors would like to thank Dr. D. Keiswetter and Dr. I. J. Won for their assistance with the GEM-3. They would also like to thank R. Weaver for supplying the landmines and for his support of this work through the Joint UXO Coordinate Office
(JUXOCO), Fort Belvoir, VA. They would also like to thank the reviewers, Y. Tan for her assistance with the data collection, and Dr. L. Nolte and Dr. S. Tantum for useful discussions regarding this work.

\section{REFERENCES}

[1] L. Collins, P. Gao, and L. Carin, "An improved bayesian decision theoretic approach for land mine detection," IEEE Trans. Geosci. Remote Sensing, vol. 37, pp. 811-819, Mar. 1999.

[2] C. E. Baum, "Low Frequency Near-Field Magnetic Scattering from Highly, but Not Perfectly Conducting Bodies," Phillips Laboratory, Interaction Note 499, Nov. 1993.

[3] G. D. Sower and S. P. Cave, "Detection and identification of mines from natural magnetic and electromagnetic resonances," in Proc. SPIE, Orlando, FL, 1995.

[4] A. H. Trang, P. V. Czipott, and D. A. Waldron, "Characterization of small metallic objects and non-metallic anti-personnel mines," in Proc. SPIE, Orlando, FL, 1997.

[5] S. Vitebskiy and L. Carin, "Short-pulse plane wave scattering from a buried perfectly conducting body of revolution," IEEE Trans. Antennas Propagat., vol. 44, pp. 112-120, Jan. 1996.

[6] S. Vitebskiy and L. Carin, "Late-time resonant frequencies of buried bodies of revolution," IEEE Trans. Antennas Propagat., vol. 44, pp. 1575-1583, Dec. 1996.

[7] C. E. Baum, N. Geng, and L. Carin, "Integral Equations and Polarizability for Magnetic Singularity Identification," Phillips Lab., Interaction Note 524, Mar. 1997.

[8] N. Geng, C. E. Baum, and L. Carin, "On the low-frequency natural response of conducting and permeable targets," IEEE Trans. Geosci. Remote Sensing, vol. 37, pp. 347-359, Jan. 1999.

[9] I. J. Won, D. A. Keiswetter, and D. R. Hansen, "GEM-3: A monostatic broadband electromagnetic induction sensor," J. Env. Eng. Geophys., vol. 2, pp. 53-64, Aug. 1997.

[10] D. Keiswetter, I. J. Won, B. Barrow, and T. Bell, Object Identification Using Multifrequency EMI Data, UXO Forum, Atlanta, Georgia, May 1999.

[11] P. Gader, H. Frigui, G. Vaillette, B. Nelson, and J. Keller, "New results in fuzzy set based detection of landmines with GPR," in Detection and Remediation Technologies for Mines and Minelike Targets IV Conf., Int. Symp. Aerospace/Defense Sensing and Controls, Orlando, FL, Apr. 1999.

[12] N. Geng et al., "Wideband Electromagnetic Induction for Metal-Target Identification: Theory, Measurement and Signal Processing," Duke Univ., Technical Report, Sept. 1997.

[13] I. J. Won, D. Keiswetter, G. R. A. Fields, and L. C. Sutton, "GEM-2: A new multifrequency electromagnetic sensor," J. Env. Eng. Geophys., vol. 1, pp. 129-137, Mar. 1996.

[14] R. F. Harrington, Field Computation by Moment Methods. New York: Macmillan, 1968.

[15] J. R. Mautz and R. F. Harrington, "Radiation and scattering from bodies of revolution," Appl. Sci. Res., vol. 20, pp. 405-435, June 1969.

[16] T. Wu and L. L. Tsai, "Scattering from arbitrarily-shaped lossy dielectric bodies of revolution," Radio Sci., vol. 12, pp. 709-718, Sept. 1977.

[17] J. R. Mautz and R. F. Harrington, "Electromagnetic scattering from a homogeneous material body of revolution," $A E \ddot{U}$, vol. 33 , pp. 71-80, Feb. 1979.

[18] A. W. Glisson and D. R. Wilton, "Simple and efficient numerical methods for problems of electromagnetic radiation and scattering from surfaces," IEEE Trans. Antennas Propagat., vol. 28, pp. 593-603, Sept. 1980.

[19] A. W. Glisson, D. Kajfez, and J. James, "Evaluation of modes in dielectric resonators using a surface integral equation formulation," IEEE Trans. Microw. Theory Technol., vol. 31, pp. 1023-1029, Dec. 1983.

[20] A. W. Glisson, "Integral equation techniques," in Dielectric Resonators, D. Kajfez and P. Guillon, Eds. Dedham, MA: Artech House, 1986, pp. 259-325.

[21] C. A. Balanis, Antenna Theory-Analysis and Design. New York: Wiley, 1982.

[22] K. Simonyi, Theoretische Elektrotechnik. Berlin, Germany: SpringerVerlag, 1980

[23] C. A. Balanis, Advanced Engineering Electromagnetics: Wiley, 1989.

[24] J. C. Hancock and P. A. Wintz, Signal Detection Theory. New York: McGraw Hill, 1966, pp. 80-84.

[25] H. L. Van Trees, Detection, Estimation, and Modulation Theory. New York: Wiley, 1968. 
[26] R. J. Schalkoff, Pattern Recognition: Statistical, Structural and Neural Approaches. New York: Wiley, 1992, pp. 34-43.

[27] K. Fukunaga, Introduction to Statistical Pattern Recognition, 2nd ed. San Diego, CA: Academic, 1990, p. 127.

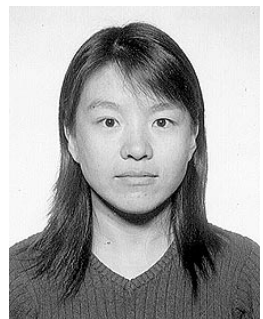

Ping Gao (S'97) received the B.S. and M.E. degrees (honors) in electrical engineering from Beijing University of Posts and Telecommunications, Beijing, China, in 1993 and 1996, respectively, and the M.S.E.E. from Duke University, Durham, NC, in 1997, where she is currently pursuing the Ph.D. degree in the Department of Electrical and Computer Engineering.

Since 1997, she has worked as a Research Assistant at Duke University. Her main research interests are in signal processing, detection and estimation theory, statistical signal modeling and analysis, and applied electromagnetics.

Leslie Collins (M'96) was born in 1963 in Raleigh, NC. She received the B.S.E.E. degree from the University of Kentucky, Lexington, in 1985, and the M.S.E.E. and Ph.D. degrees in electrical engineering, both from the University of Michigan, Ann Arbor, in 1986 and 1995, respectively.

She was a Senior Engineer with the Westinghouse Research and Development Center, Pittsburgh, PA, from 1986 to 1990. In 1995, she became an Assistant Professor in the Electrical and Computer Engineering Department, Duke University, Durham, NC. Her current research interests include incorporating physics-based models into statistical signal processing algorithms, and she is pursuing applications in subsurface sensing as well as enhancing speech understanding by hearing impaired individuals.

Dr. Collins is a member of the Tau Beta Pi, Eta Kappa Nu, and Sigma Xi honor societies.
Philip M. Garber (S'99) was born in Harrisburg, PA, in 1976. He received the B.S. degree in electrical engineering from Duke University, Durham, NC, in 1999.

He is currently a Graduate Student with the Radiation Laboratory, University of Michigan, Ann Arbor. His interests are in microwave circuits, communications, and wireless systems.

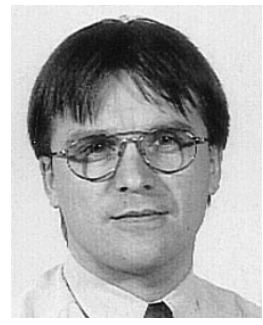

Norbert Geng (S'91-M'96) was born May 14, 1965 in Lauchringen, Germany. He received the Dipl.-Ing. and Dr.-Ing. degrees in electrical engineering from the University of Karlsruhe, Germany in 1991 and 1996, respectively.

From 1991 to 1996, he was with the Institute for Microwaves and Electronics, University of Karlsruhe, working on full-wave propagation modeling for radio communication systems. In January 1997, he joined the Department of Electrical and Computer Engineering, Duke University, Durham, NC, as a Visiting Postdoctoral Fellow for 18 months. Since July 1998, he has been with the University of Karlsruhe. His current research interests include computational methods in electromagnetics and wave propagation modeling.

Dr. Geng received the Mannesmann Innovation Award in 1997 for his Ph.D thesis on full-wave propagation modeling for radio communication systems.

Lawrence Carin (SM'96) was born on March 25, 1963, in Washington, DC, and earned the B.S., M.S., and Ph.D. degrees in electrical engineering at the University of Maryland, College Park, in 1985, 1986, and 1989, respectively.

In 1989, he joined the Electrical Engineering Department, Polytechnic University, New York, as an Assistant Professor, and became an Associate Professor there in 1994. In September 1995, he joined the Electrical Engineering Department, Duke University, Durham, NC, where he is an Associate Professor. His current research interests include short pulse scattering, subsurface sensing, and wave-based signal processing.

Dr. Carin is a member of the Tau Beta Pi and Eta Kappa Nu honor societies. $\mathrm{He}$ is currently an Associate Editor of the IEEE TRANSACTIONS ON ANTENNAS AND PROPAGATION. 